\title{
Noncommutative spectral geometry: A guided tour for theoretical physicists
}

\author{
Mairi Sakellariadou* \\ Department of Physics, King's College London, University of London, Strand WC2R 2LS, \\ London, U.K. \\ E-mail: Mairi.Sakellariadou@kcl.ac.uk
}

\begin{abstract}
We review a gravitational model based on noncommutative geometry and the spectral action principle. The space-time geometry is described by the tensor product of a four-dimensional Riemanian manifold by a discrete noncommutative space consisting of only two points. With a specific choice of the finite dimensional involutive algebra, the noncommutative spectral action leads to the standard model of electroweak and strong interactions minimally coupled to Einstein and Weyl gravity. We present the main mathematical ingredients of this model and discuss their physical implications. We argue that the doubling of the algebra is intimately related to dissipation and the gauge field structure. We then show how this noncommutative spectral geometry model, a purely classical construction, carries implicit in the doubling of the algebra the seeds of quantization. After a short review on the phenomenological consequences of this geometric model as an approach to unification, we discuss some of its cosmological consequences. In particular, we study deviations of the Friedmann equation, propagation of gravitational waves, and investigate whether any of the scalar fields in this model could play the rôle of the inflaton.
\end{abstract}

\footnotetext{
Proceedings of the Corfu Summer Institute 2011 "School and Workshops on Elementary Particle Physics and Gravity"

September 4-18, 2011

Corfu, Greece
}

\footnotetext{
*Speaker.
} 


\section{Introduction}

One of the main open issues in theoretical high energy physics is the unification of all forces, including gravity, so that all interactions correspond to one underlying symmetry. At low energy scales, one can consider an effective theory with physics being described by the sum of the EinsteinHilbert and the Standard Model action. However, while the first part of this action is based upon diffeomorphism invariance, the second one is based upon internal symmetries of a gauge group. This different nature of symmetries for the two parts of the effective action may be at the origin to the difficulty of finding a unified theory of all interactions, including gravity. As one approaches the Planck energy scale the quantum nature of space-time reveals itself and this simplistic effective theory breaks down. Close to the Planck scale the appropriate formulation of geometry should be within a quantum framework and the nature of space-time would change in a way so that one can recover the low energy picture of diffeomorphism and internal gauge symmetries. A proposal that could lead to a quantum nature of space-time has been introduced within noncommutative geometry.

In the framework of noncommutative spectral geometry, gravity and the standard model fields were put together into matter and geometry on a noncommutative space made from the product of a four-dimensional standard commutative manifold by a noncommutative internal space. The approach is based on a simple idea: using a very simple mathematical input, namely the choice of a finite dimensional algebra, one can derive [1] the full complexity of the standard model Lagrangian coupled to gravity, by employing the formalism of noncommutative geometry and spectral action.

Noncommutative spectral geometry offers an elegant approach to unification, based on the symplectic unitary group in Hilbert space, rather than on finite dimensional Lie groups. The model offers a unification of internal symmetries with the gravitational ones. All symmetries arise as automorphisms of the noncommutative algebra of coordinates on a product geometry. Due to the lack of a full quantum gravity theory, which a priori should define the geometry of spacetime at Planckian energy scales, we will follow an effective theory approach and consider the simplest case beyond commutative spaces. Thus, below but close to the Planck energy scale, spacetime will be considered as the product of a Riemanian spin manifold by a finite noncommutative space. At higher energy scales space-time should become noncommutative in a nontrivial way, while at energies above the Planck scale the whole concept of geometry may altogether become meaningless. As a next but highly nontrivial step, one should consider noncommutative spaces whose limit is the almost commutative space considered here.

It is worth clarifying that the noncommutative spectral geometry approach discussed here, goes beyond the noncommutative geometry notion employed in the literature to implement the fuzziness of space-time by means of $\left[\mathbf{x}^{i}, \mathbf{x}^{j}\right]=i \theta^{i j}$, where $\theta^{i j}$ is an anti-symmetric, real, $d \times d$ ( $d$ stands for the dimension of space-time) matrix, and $\mathbf{x}^{i}$ denote spatial coordinates.

In what follows, we briefly present the elements of noncommutative spectral geometry [2,3] as an approach to unification and highlight the relation between the doubling of the algebra and the gauge fields [4], an essential element to make the link with the standard model of particle physics. We then argue that the doubling of the algebra is related to dissipation, which incorporates the seeds of quantization [4]. After a short review on the phenomenological predictions of this purely geometric approach to the standard model, we discuss some of its cosmological consequences [5, 
$6,7,8,9,10]$.

\section{Elements of noncommutative spectral geometry}

We consider the geometry of space-time as being described by the tensor product $\mathscr{M} \times \mathscr{F}$ of a four-dimensional smooth compact ${ }^{1}$ Riemanian manifold $\mathscr{M}$ by a tiny discrete finite noncommutative space $\mathscr{F}$ composed of just two points. The geometry is thus described by the product of a continuous geometry for space-time by an internal geometry for the standard model of particle physics. The finite geometry $\mathscr{F}$ will be chosen so that it is one of the simplest and most natural finite noncommutative geometries of the right dimension to solve the fermion doubling problem.

The noncommutative nature of the finite discrete space $\mathscr{F}$ is given by the spectral triple $\left(\mathscr{A}_{\mathscr{F}}, \mathscr{H}_{\mathscr{F}}, \mathscr{D}_{\mathscr{F}}\right)$, where all ingredients are finite dimensional. In the spectral triple, $\mathscr{A}_{\mathscr{F}}$ is an involution of operators on the finite-dimensional Hilbert space $\mathscr{H}_{\mathscr{F}}$ of Euclidean fermions and $\mathscr{D}_{\mathscr{F}}$ a self-adjoint unbounded operator in $\mathscr{H}_{\mathscr{F}}$. The operator $\mathscr{D}_{\mathscr{F}}$ is such that $J \mathscr{D}_{\mathscr{F}}=\varepsilon^{\prime} \mathscr{D}_{\mathscr{F}} J$, where $J$ is an anti-linear isometry of the finite dimensional Hilbert space, with the properties

$$
J^{2}=\varepsilon, J \gamma=\varepsilon^{\prime \prime} \gamma J
$$

$\gamma$ is the chirality operator and $\varepsilon, \varepsilon^{\prime}, \varepsilon^{\prime \prime} \in\{ \pm 1\}$.

Let us discuss the physical reason for introducing the discrete space $\mathscr{F}$. There is a distinction between the metric (spectral) dimension, specified by the behavior of the eigenvalues of the Dirac operator, and the KO-dimension (K-theoretic dimension), an algebraic dimension based on K-theory. We first start with the metric dimension. The relevant Dirac operator for space-time is the ordinary Dirac operator on a curved space-time, thus the metric dimension is equal to four. The internal Dirac operator consists of the fermionic mass matrix, which has a finite number of eigenvalues, and therefore the internal metric dimension is equal to zero. Thus, the metric dimension of the $\mathscr{M} \times \mathscr{F}$ geometry is just four, the same as that of the ordinary space-time manifold. We proceed with the KO-dimension. There are 8 possible combinations for the numbers $\varepsilon, \varepsilon^{\prime}, \varepsilon^{\prime \prime}$, leading to a KO-dimension modulo 8 . To resolve the fermion doubling problem, by projecting out the unphysical degrees of freedom resting in the internal space, the real structure of the finite geometry $\mathscr{F}$ turns out to be such that its KO-dimension is equal to six, leading to $\left(\varepsilon, \varepsilon^{\prime}, \varepsilon^{\prime \prime}\right)=(1,1,-1)$. Setting the KO-dimension of the product space $\mathscr{M} \times \mathscr{F}$ to be $10 \sim 2$ modulo 8 , allows one to impose simultaneously the reality and Weyl conditions in the Minkowskian continued forms. Thus, the reason for introducing $\mathscr{F}$ is to correct the KO-dimension from four to ten (modulo 8). In other words, the fermion doubling problem requires $[11,12]$ crossing the ordinary four-dimensional continuum by a space of KO-dimension 6.

The spectral geometry is given by the product rules:

$$
\mathscr{A}=C^{\infty}(\mathscr{M}) \oplus \mathscr{A}_{\mathscr{F}}, \quad \mathscr{H}=L^{2}(\mathscr{M}, S) \oplus \mathscr{H}_{\mathscr{F}}, \mathscr{D}=\mathscr{D} \mathscr{M} \oplus 1+\gamma_{5} \oplus \mathscr{D} \mathscr{F}
$$

where $L^{2}(\mathscr{M}, S)$ is the Hilbert space of $L^{2}$ spinors and $\mathscr{D} \mathscr{M}$ is the Dirac operator of the Levi-Civita spin connection on the four-dimensional manifold $\mathscr{M}$. The chirality operator is $\gamma=\gamma_{5} \oplus \gamma_{\mathscr{F}}$ and the

\footnotetext{
${ }^{1}$ The Euclidean space-time manifold is taken to be compact for simplicity.
} 
anti-unitary operator on the complex Hilbert space is $J=J_{\mathscr{M}} \oplus J_{\mathscr{F}}$, with $J_{\mathscr{M}}$ the charge conjugation. In order to avoid the fermion doubling problem, one must take

$$
J_{\mathscr{F}}^{2}=1, J_{\mathscr{F}} \mathscr{D}_{\mathscr{F}}=\mathscr{D}_{\mathscr{F}} J_{\mathscr{F}}, J_{\mathscr{F}} \gamma_{\mathscr{F}}=-\gamma_{\mathscr{F}} J_{\mathscr{F}}
$$

In what follows we only consider the noncommutative discrete space $\mathscr{F}$; to simplify the notation we omit the subscript $\mathscr{F}$.

The main input of this purely geometric approach to the standard model of particle physics is the choice of a finite dimensional involutive algebra [2,3]. In the context of left-right symmetric models, the algebra is a direct sum of the matrix algebras $M_{N}(\mathbb{C})$ with $N=1,3$ with two copies $\mathbb{H}_{L}, \mathbb{H}_{R}$ of the algebra of quaternions $\mathbb{H}$, namely

$$
\mathscr{A}_{\mathrm{LR}}=\mathbb{C} \oplus \mathbb{H}_{\mathrm{L}} \oplus \mathbb{H}_{\mathrm{R}} \oplus M_{3}(\mathbb{C}) ;
$$

the left-right symmetric algebra. The fermions of the standard model can be identified with a basis for a sum of 3 (i.e. the number of generations, which is considered here to be equal to 3 ) copies of the representation of the algebra $\mathscr{A}_{\mathrm{LR}}$, which is the sum of the irreducible bimodules of odd spin. The algebra $\mathscr{A}_{\mathrm{LR}}$ admits a natural sub-algebra $\mathbb{C} \oplus M_{3}(\mathbb{C})$, corresponding to integer spin.

However, our aim is to construct a model that accounts for massive neutrinos and neutrino oscillations, thus it cannot be a left-right symmetric model. We will therefore select a sub-algebra of the left-right symmetric algebra, which breaks left-right symmetry and leads to the involutive algebra:

$$
\left\{\left(\lambda, q_{\mathrm{L}}, \lambda, m\right) \mid \lambda \in \mathbb{C}, q_{\mathrm{L}} \in \mathbb{H}, m \in M_{3}(\mathbb{C})\right\},
$$

isomorphic to $\mathbb{C} \oplus \mathbb{H} \oplus M_{3}(\mathbb{C})$. The algebra of quaternions ${ }^{2} \mathbb{H} \subset M_{2}(\mathbb{C})$ is

$$
\mathbb{H}=\left\{\left(\begin{array}{cc}
\alpha & \beta \\
-\bar{\beta} & \bar{\alpha}
\end{array}\right) ; \alpha, \beta \in \mathbb{C}\right\} .
$$

Consider a finite dimensional Hilbert space $\mathscr{H}$ of dimension $n$, with an anti-unitary operator $J$, such that $J^{2}=1$. Noncommutative geometry imposes constraints on the involutive algebras of operators in the Hilbert space. The involutive algebra $\mathscr{A}$ must be such that

$$
\left[a, b^{0}\right]=0, \forall a, b \in \mathscr{A},
$$

where $b^{0}=J b^{\star} J^{-1}$ and the representation of $\mathscr{A}$ and $J$ in $\mathscr{H}$ is an irreducible representation. To get an irreducible solution, the dimension $n$ must be either $k^{2}$ or $2 k^{2}$. Classifying all irreducible finite noncommutative geometries of KO-dimension six, it was shown [13] that only $n=2 k^{2}$ can avoid fermion doubling. There are thus six possibilities for the algebra $\mathscr{A}$, namely

$$
\left\{M_{k}(\mathbb{C}) \text { or } M_{k}(\mathbb{R}) \text { or } M_{a}(\mathbb{H})\right\} \oplus\left\{M_{k}(\mathbb{C}) \text { or } M_{k}(\mathbb{R}) \text { or } M_{a}(\mathbb{H})\right\} \text {. }
$$

It turns out that five of these possibilities are ruled out [14]. Imposing an anti-linear isometry $I$ such that $I^{2}=-1$ in just one of the algebras and letting the other one free, the algebra $\mathscr{A}$ must be then the following one [15]:

$$
\mathscr{A}=M_{a}(\mathbb{H}) \oplus M_{k}(\mathbb{C}) \quad \text { with } \quad k=2 a .
$$

\footnotetext{
${ }^{2}$ To obtain the Lagrangian of the standard model of particle physics we assume quaternion linearity.
} 
The choice $k=4$ is the first one that produces the correct number of $k^{2}=16$ fermions in each of the three generations ${ }^{3}$; the number of generations is a physical input.

For commutative geometries, a real variable described by a real-valued function on a space is given by the corresponding algebra of coordinates, while for noncommutative geometries is represented as operators in a fixed Hilbert space. Since real coordinates are represented by selfadjoint operators, all information about space is encoded in the algebra of coordinates $\mathscr{A}$, which is related to the gauge group of local gauge transformations. While the choice of the algebra constitutes the main input of this model, the choice of Hilbert space is irrelevant.

The operator $\mathscr{D}$ corresponds to the inverse of the Euclidean propagator of fermions. It is given by the Yukawa coupling matrix which encodes the masses of the elementary fermions and the Kobayashi-Maskawa mixing parameters. The commutator $[D, a]$, with $a \in \mathscr{A}$, plays the rôle of the differential quotient $d a / d s$, with $d s$ the unit of length. The familiar geodesic formula

$$
d(x, y)=\inf \int_{\gamma} d s
$$

(the infimum is taken over all possible paths connecting $x$ to $y$ ), which is used in Riemanian geometry to determine the distance $d(x, y)$ between two points $x$ and $y$, is replaced in noncommutative geometry by

$$
d(x, y)=\sup \{|f(x)-f(y)|: f \in \mathscr{A},\|[D, f]\| \leq 1\}
$$

(where $D$ is the inverse of the line element $d s$ ). To describe noncommutative geometry, we will focus on the Dirac operator $\mathscr{D}$, instead of the metric tensor $g_{\mu v}$ which is used for spaces with commuting coordinates. The standard model fermions provide the Hilbert space $\mathscr{H}$ of a spectral triple for the algebra $\mathscr{A}$, while the bosons are obtained through inner fluctuations of the Dirac operator of the product geometry.

Since all experimental data are of a spectral nature, we aim at extracting information, from our noncommutative geometry construction, which is of a spectral nature. The spectral action functional in noncommutative spaces is analogous to the Fourier transform in spaces for which spatial coordinates commute. We then apply the spectral action principle, stating that the bare bosonic ${ }^{4}$ Euclidean action is the trace of the heat kernel associated with the square of the Dirac operator and is of the form $\operatorname{Tr}(f(\mathscr{D} / \Lambda)) ; f$ is a cut-off function and $\Lambda$ fixes the energy scale. This action can be seen à la Wilson as the bare action at energy scale $\Lambda$. Thus, following the Wilsonian approach, one can obtain physical predictions for the standard model parameters by running them down to low (present) energy scales through the renormalization group equations. Let us emphasize that this picture is only valid at high energies (at the scale $\Lambda$, taken to be the unification scale) and the spectral action must be considered in the Wilsonian approach, where all coupling constants are energy dependent and follow the renormalization group equations. Since both $\mathscr{D}$ and $\Lambda$ have physical dimensions of a mass, there is no absolute scale on which they can be measured. The rôle of the cut-off scale $\Lambda$ is equivalent to keeping only frequencies smaller than the mass scale $\Lambda$. Note

\footnotetext{
${ }^{3}$ If at the CERN Large Hadron Collider new particles are discovered, one may be able to include them by considering a higher value for $k$.

${ }^{4}$ The fermionic term can be included by adding $(1 / 2)\langle J \psi, \mathscr{D} \psi\rangle$, where $J$ is the real structure on the spectral triple and $\psi$ is a spinor in the Hilbert space of the quarks and leptons.
} 
that $\operatorname{Tr}(f(\mathscr{D} / \Lambda))$ is the fundamental action functional that can be used not only at the classical level but also at the quantum level, after Wick rotation to Euclidean signature.

The formalism of spectral triples favors Euclidean rather than Lorentzian signature ${ }^{5}$. The discussion of phenomenological/cosmological aspects of the theory relies on a Wick rotation to imaginary time, into the Lorentzian signature. While sensible from the phenomenological point of view, there exists as yet no justification on the level of the underlying theory.

In conclusion, one can obtain the full standard model minimally coupled to Einstein and Weyl gravity, plus higher order nonrenormalizable interactions suppressed by powers of the inverse of the mass scale of the theory, through the action functional [1]

$$
\mathscr{S}=\operatorname{Tr}\left(f\left(\frac{\mathscr{D}}{\Lambda}\right)\right)+\frac{1}{2}\langle J \psi, D \psi\rangle, \psi \in \mathscr{H}^{+}
$$

applied to uni-modular inner fluctuations

$$
\mathscr{D} \rightarrow \mathscr{D}_{A}=\mathscr{D}+A+\varepsilon^{\prime} J A J^{-1}
$$

$A=A^{\star}$ is a self-adjoint operator of the form

$$
A=\sum_{j} a_{j}\left[\mathscr{D}, b_{j}\right], a_{j}, b_{j} \in \mathscr{A} .
$$

Using heat kernel methods, the trace $\operatorname{Tr}\left(f\left(\mathscr{D}_{A} / \Lambda\right)\right)$ can be written in terms of the geometrical Seeley-de Witt coefficients $a_{n}$, which are known for any second order elliptic differential operator, as $\sum_{n=0}^{\infty} F_{4-n} \Lambda^{4-n} a_{n}$, where the function $F$ is defined such that $F\left(\mathscr{D}_{A}^{2}\right)=f\left(\mathscr{D}_{A}\right)$. Thus, the bosonic part of the spectral action can be expanded in powers of $\Lambda$ in the form [16, 17]

$$
\operatorname{Tr}\left(f\left(\frac{\mathscr{D}_{A}}{\Lambda}\right)\right) \sim \sum_{k \in \operatorname{DimSp}} f_{k} \Lambda^{k} f\left|\mathscr{D}_{A}\right|^{-k}+f(0) \zeta_{\mathscr{D}_{A}(0)}+\mathscr{O}(1) .
$$

The momenta $f_{k}$ are defined as $f_{k} \equiv \int_{0}^{\infty} f(u) u^{k-1} \mathrm{~d} u$ for $k>0$ and $f_{0} \equiv f(0)$, the noncommutative integration is defined in terms of residues of zeta functions $\zeta_{\mathscr{D}_{A}}(s)=\operatorname{Tr}\left(\left|\mathscr{D}_{A}\right|^{-s}\right)$ at poles of the zeta function, and the sum is over points in the dimension spectrum of the spectral triple.

For the four-dimensional Riemanian geometry, the trace is expressed perturbatively in terms of the geometrical Seeley-deWitt coefficients $a_{n}$, as [18]:

$$
\operatorname{Tr}\left(f\left(\frac{\mathscr{D}_{A}}{\Lambda}\right)\right) \sim 2 \Lambda^{4} f_{4} a_{0}+2 \Lambda^{2} f_{2} a_{2}+f_{0} a_{4}+\cdots+\Lambda^{-2 k} f_{-2 k} a_{4+2 k}+\cdots
$$

The smooth even function $f$, which decays fast at infinity, only enters in the multiplicative factors:

$$
\begin{aligned}
f_{4} & =\int_{0}^{\infty} f(u) u^{3} d u, \\
f_{2} & =\int_{0}^{\infty} f(u) u d u, \\
f_{0} & =f(0), \\
f_{-2 k} & =(-1)^{k} \frac{k !}{(2 k) !} f^{(2 k)}(0) .
\end{aligned}
$$

\footnotetext{
${ }^{5}$ The issue of Euclidean versus Lorentzian signature is also encountered in the nonperturbative path-integral approach to quantum gravity.
} 
Since $f$ is taken as a cut-off function, its Taylor expansion at zero vanishes, therefore the asymptotic expansion, Eq. (2.6), reduces to

$$
\operatorname{Tr}\left(f\left(\frac{\mathscr{D}_{A}}{\Lambda}\right)\right) \sim 2 \Lambda^{4} f_{4} a_{0}+2 \Lambda^{2} f_{2} a_{2}+f_{0} a_{4}
$$

the cut-off function $f$ plays a rôle only through its momenta $f_{0}, f_{2}, f_{4}$, which are three real parameters, related to the coupling constants at unification, the gravitational constant, and the cosmological constant, respectively. In the four-dimensional case, the term in $\Lambda^{4}$ in the spectral action, Eq. (2.5), gives a cosmological term, the term in $\Lambda^{2}$ gives the Einstein-Hilbert action functional with the physical sign for the Euclidean functional integral (provided $f_{2}>0$ ), and the $\Lambda$-independent term yields the Yang-Mills action for the gauge fields corresponding to the internal degrees of freedom of the metric. The scale-independent terms in the spectral action have conformal invariance.

The physical Lagrangian, obtained by applying the spectral action principle in the product geometry, is entirely determined by the geometric input, namely the $\mathscr{M} \times \mathscr{F}$ space. It contains, in addition to the full standard model Lagrangian, the Einstein-Hilbert action with a cosmological term, a topological term related to the Euler characteristic of the space-time manifold, a conformal Weyl term and a conformal coupling of the Higgs field to gravity. The Higgs field is the vector boson of the internal noncommutative degrees of freedom. The bosonic action in Euclidean signature reads [1]

$$
\begin{array}{r}
\mathscr{S}^{\mathrm{E}}=\int\left(\frac{1}{2 \kappa_{0}^{2}} R+\alpha_{0} C_{\mu v \rho \sigma} C^{\mu v \rho \sigma}+\gamma_{0}+\tau_{0} R^{\star} R^{\star}+\frac{1}{4} G_{\mu v}^{i} G^{\mu v i}+\frac{1}{4} F_{\mu v}^{\alpha} F^{\mu v \alpha}\right. \\
\left.+\frac{1}{4} B^{\mu v} B_{\mu v}+\frac{1}{2}\left|D_{\mu} \mathbf{H}\right|^{2}-\mu_{0}^{2}|\mathbf{H}|^{2}-\xi_{0} R|\mathbf{H}|^{2}+\lambda_{0}|\mathbf{H}|^{4}\right) \sqrt{g} d^{4} x
\end{array}
$$

where

$$
\begin{aligned}
\kappa_{0}^{2} & =\frac{12 \pi^{2}}{96 f_{2} \Lambda^{2}-f_{0} \mathfrak{c}} \\
\alpha_{0} & =-\frac{3 f_{0}}{10 \pi^{2}} \\
\gamma_{0} & =\frac{1}{\pi^{2}}\left(48 f_{4} \Lambda^{4}-f_{2} \Lambda^{2} \mathfrak{c}+\frac{f_{0}}{4} \mathfrak{d}\right) \\
\tau_{0} & =\frac{11 f_{0}}{60 \pi^{2}} \\
\mu_{0}^{2} & =2 \Lambda^{2} \frac{f_{2}}{f_{0}}-\frac{\mathfrak{e}}{\mathfrak{a}} \\
\xi_{0} & =\frac{1}{12} \\
\lambda_{0} & =\frac{\pi^{2} \mathfrak{b}}{2 f_{0} \mathfrak{a}^{2}}
\end{aligned}
$$

$\mathbf{H}$ is a rescaling

$$
\mathbf{H}=\left(\sqrt{a f_{0}} / \pi\right) \phi,
$$

of the Higgs field $\phi$ to normalize the kinetic energy, and the momentum $f_{0}$ is physically related to the coupling constants at unification. 
Notice the absence of quadratic terms in the curvature; there is only the term quadratic in the Weyl curvature and the topological term $R^{\star} R^{\star}$. In a cosmological setting, namely for FriedmannLemaitre-Robertson-Walker geometries, the Weyl term vanishes. Notice also the term that couples gravity with the standard model, a term which should always be present when one considers gravity coupled to scalar fields. It is important to emphasize that the relations in Eq. (2.10) are tied to the scale at which the expansion is performed. There is a priori no reason for the constraints to hold at scales below the unification scale $\Lambda$, since they represent mere boundary conditions ${ }^{6}$.

The geometric parameters $\mathfrak{a}, \mathfrak{b}, \mathfrak{c}, \mathfrak{d}, \mathfrak{e}$ describe the possible choices of Dirac operators on the finite noncommutative space. These parameters correspond to the Yukawa parameters of the particle physics model and the Majorana terms for the right-handed neutrinos. They are given by [1]

$$
\begin{aligned}
\mathfrak{a} & =\operatorname{Tr}\left(Y_{(\uparrow 1)}^{\star} Y_{(\uparrow 1)}+Y_{(\downarrow 1)}^{\star} Y_{(\downarrow 1)}+3\left(Y_{(\uparrow 3)}^{\star} Y_{(\uparrow 3)}+Y_{(\downarrow 3)}^{\star} Y_{(\downarrow 3)}\right)\right), \\
\mathfrak{b} & =\operatorname{Tr}\left(\left(Y_{(\uparrow 1)}^{\star} Y_{(\uparrow 1)}\right)^{2}+\left(Y_{(\downarrow 1)}^{\star} Y_{(\downarrow 1)}\right)^{2}+3\left(Y_{(\uparrow 3)}^{\star} Y_{(\uparrow 3)}\right)^{2}+3\left(Y_{(\downarrow 3)}^{\star} Y_{(\downarrow 3)}\right)^{2}\right), \\
\mathfrak{c} & =\operatorname{Tr}\left(Y_{R}^{\star} Y_{R}\right) \\
\mathfrak{d} & =\operatorname{Tr}\left(\left(Y_{R}^{\star} Y_{R}\right)^{2}\right), \\
\mathfrak{e} & =\operatorname{Tr}\left(Y_{R}^{\star} Y_{R} Y_{(\uparrow 1)}^{\star} Y_{(\uparrow 1)}\right),
\end{aligned}
$$

with $Y_{(\downarrow 1)}, Y_{(\uparrow 1)}, Y_{(\downarrow 3)}, Y_{(\uparrow 3)}$ and $Y_{R}$ being $(3 \times 3)$ matrices, with $Y_{R}$ symmetric. The $Y$ matrices are used to classify the action of the Dirac operator and give the fermion and lepton masses, as well as lepton mixing, in the asymptotic version of the spectral action. The Yukawa parameters run with the renormalization group equations of the particle physics model.

It is worth noting that since running towards lower energies implies that nonperturbative effects in the spectral action cannot be any longer neglected, any results based on the asymptotic expansion and on renormalization group analysis can only hold for early universe cosmology. Hence, the spectral action at the unification scale $\Lambda$ offers a framework to investigate early universe cosmological models. For later times, one should consider the full spectral action, a direction which requires the development of nontrivial mathematical tools.

\section{Dissipation and the origin of quantization}

The central ingredient of the noncommutative spectral geometry model, namely the doubling of the algebra acting on the doubled Hilbert space, is also present in the quantum mechanics formalism of the Wigner function

$$
W(p, x, t)=\frac{1}{2 \pi \hbar} \int \Psi^{*}\left(x-\frac{1}{2} y, t\right) \Psi\left(x+\frac{1}{2} y, t\right) e^{-i \frac{p y}{\hbar}} d y
$$

and the density matrix

$$
W\left(x_{+}, x_{-}, t\right) \equiv\left\langle x_{+}|\rho(t)| x_{-}\right\rangle=\Psi^{*}\left(x_{-}, t\right) \Psi\left(x_{+}, t\right) .
$$

\footnotetext{
${ }^{6}$ One can find in the literature the unjustified ansatz that these boundary conditions are functions of the energy scale.
} 
Here we split the coordinate $x(t)$ of a quantum particle being into two coordinates $x_{+}(t)$ and $x_{-}(t)$ :

$$
x_{+}(t)=x(t)+\frac{1}{2} y(t) \quad \text { and } \quad x_{-}(t)=x(t)-\frac{1}{2} y(t)
$$

going forward and backward in time, respectively.

The forward and backward in time evolution of the density matrix $W\left(x_{+}, x_{-}, t\right)$ is then described by two copies of the Schrödinger equation, as

$$
i \hbar \frac{\partial\left\langle x_{+}|\rho(t)| x_{-}\right\rangle}{\partial t}=H\left\langle x_{+}|\rho(t)| x_{-}\right\rangle,
$$

where $H=H_{+}-H_{-}$with $H_{ \pm}$the two Hamiltonian operators.

Thus, the density matrix and the Wigner function require the introduction of a doubled set of coordinates and of their respective algebras. Equation (3.3) implies that the eigenvalues of $H$ are directly the Bohr transition frequencies

$$
h v_{n m}=E_{n}-E_{m}
$$

which was the first hint towards an explanation of spectroscopic structure. This can be seen as the connection between noncommutative algebra, spectroscopic experiments and energy level discretization.

Moreover, the doubling of the algebra is implicit even in the classical theory when considering the Brownian motion of a particle with equation of motion

$$
m \ddot{x}(t)+\gamma \dot{x}(t)=f(t)
$$

$f(t)$ is a random Gaussian distributed force with

$$
<f(t) f\left(t^{\prime}\right)>_{\text {noise }}=2 \gamma k_{B} T \delta\left(t-t^{\prime}\right) .
$$

Equation (3.4) can be derived [19] from a Lagrangian in a canonical procedure, using a delta functional classical constraint representation as a functional integral. By averaging over the fluctuating force $f$, one gets

$$
<\delta[m \ddot{x}+\gamma \dot{x}-f]>_{\text {noise }}=\int D y<\exp \left[\frac{i}{\hbar} \int d t L_{f}(\dot{x}, \dot{y}, x, y)\right]>_{\text {noise }},
$$

where

$$
\mathscr{L}_{f}(\dot{x}, \dot{y}, x, y)=m \dot{x} \dot{y}+\frac{\gamma}{2}(x \dot{y}-y \dot{x})+f y .
$$

Hence, the constraint condition at the classical level introduced a new coordinate $y$, and the standard Euler-Lagrange equations are obtained:

$$
\frac{d}{d t} \frac{\partial \mathscr{L}_{f}}{\partial \dot{y}}=\frac{\partial \mathscr{L}_{f}}{\partial y} ; \quad \frac{d}{d t} \frac{\partial \mathscr{L}_{f}}{\partial \dot{x}}=\frac{\partial \mathscr{L}_{f}}{\partial x}
$$

leading to

$$
m \ddot{x}+\gamma \dot{x}=f, \quad m \ddot{y}-\gamma \dot{y}=0 .
$$


It is worth noting that the Lagrangian system, Eqs. (3.7)-(3.9), above was obtained in a completely classical context ${ }^{7}$ in order to build a canonical formalism for a dissipative system. The $x$-system is an open system; to set up the canonical formalism it is required to close it and this is done by introducing its time-reversed copy, the $y$-system. The resulting $\{x-y\}$-system is a closed one.

To highlight [4] the relation between the doubling of the algebra and the gauge field structure let us consider the equation of the classical one-dimensional damped harmonic oscillator

$$
m \ddot{x}+\gamma \dot{x}+k x=0,
$$

with time independent $m, \gamma$ and $k$. As we have just discussed, in the canonical formalism for open systems the doubling of the degrees of freedom is required in such a way as to complement the given open system with its time-reversed image, and thus obtain a globally closed system for which the Lagrangian formalism is well defined. Considering the oscillator in the doubled $y$-coordinate

$$
m \ddot{y}-\gamma \dot{y}+k y=0,
$$

and introducing the coordinates

$$
x_{1}(t)=\frac{x(t)+y(t)}{\sqrt{2}} \quad \text { and } \quad x_{2}(t)=\frac{x(t)-y(t)}{\sqrt{2}},
$$

the Lagrangian of this closed system takes the form

$$
\mathscr{L}=\frac{1}{2 m}\left(m \dot{x}_{1}+\frac{e_{1}}{c} A_{1}\right)^{2}-\frac{1}{2 m}\left(m \dot{x}_{2}+\frac{e_{2}}{c} A_{2}\right)^{2}-\frac{e^{2}}{2 m c^{2}}\left(A_{1}^{2}+A_{2}^{2}\right)-e \Phi,
$$

where we have introduced the vector potential

$$
A_{i}=\frac{B}{2} \varepsilon_{i j} x_{j} \quad \text { for } \quad i, j=1,2,
$$

with

$$
B \equiv \frac{\gamma_{c}}{e}
$$

and

$$
\varepsilon_{i i}=0 \quad, \quad \varepsilon_{12}=-\varepsilon_{21}=1 ;
$$

The Lagrangian Eq. (3.13) describes two particles with opposite charges $e_{1}=-e_{2}=e$ in the potential

$$
\Phi \equiv \frac{k}{2 e}\left(x_{1}^{2}-x_{2}^{2}\right) \equiv \Phi_{1}-\Phi_{2},
$$

with $\Phi_{i} \equiv(k / 2 / e) x_{i}{ }^{2}$, in the constant magnetic field $\mathbf{B}$ defined by $\mathbf{B}=\nabla \times \mathbf{A}$.

Thus, the doubled coordinate, e.g., $x_{2}$ acts as the gauge field component $A_{1}$ to which the $x_{1}$ coordinate is coupled, and vice versa. In conclusion, the energy dissipated by one of the two systems is gained by the other, implying that the gauge field acts as the bath or reservoir in which the system is embedded [4].

Following 't Hooft's conjecture [20], stating that there are classical deterministic models for which loss of information might lead to a quantum evolution, we argue [4] that the noncommutative

\footnotetext{
${ }^{7}$ Note that $\hbar$ has been introduced for dimensional reasons.
} 
spectral geometry classical construction carries implicit in its feature of the doubling of the algebra the seeds of quantization. We will show that the Hamiltonian of a classical damped harmonic $x$ oscillator and its time-reversed image, the $y$-oscillator, belongs to the class of Hamiltonians for which this conjecture was proposed.

The system's Hamiltonian can be written as $[21,22]$

$$
H=\sum_{i=1}^{2} p_{i} f_{i}(q)
$$

with the functions $f_{1}, f_{2}$ given by

$$
f_{1}(q)=2 \Omega \quad, \quad f_{2}(q)=-2 \Gamma,
$$

where

$$
\Gamma=\frac{\gamma}{2 m}, \Omega=\sqrt{\frac{1}{m}\left(k-\frac{\gamma^{2}}{4 m}\right)} \quad \text { with } k>\frac{\gamma^{2}}{4 m} .
$$

The nonvanishing Poisson brackets are $\left\{q_{i}, p_{i}\right\}=1$.

The Hamiltonian Eq. (3.18) belongs to the class of Hamiltonians considered by 't Hooft. The $f_{i}(q)$ are nonsingular functions of the canonical coordinates $q_{i}$ and the equations for the $q$ 's, namely $\dot{q}_{i}=\left\{q_{i}, H\right\}=f_{i}(q)$ ), are decoupled from the conjugate momenta $p_{i}$. In such a case, there is a complete set of observables which Poisson commute at all times. This implies that the system admits a deterministic description even when expressed in terms of operators acting on some functional space of states $|\Psi\rangle$, such as the Hilbert space. Such a description in terms of operators and Hilbert space, does not imply per se quantization of the system. Quantization is achieved only as a consequence of dissipation.

Let us write the Hamiltonian as

$$
H=H_{\mathrm{I}}-H_{\mathrm{II}}
$$

with

$$
H_{\mathrm{I}}=\frac{1}{2 \Omega \mathscr{C}}\left(2 \Omega \mathscr{C}-\Gamma J_{2}\right)^{2}, H_{\mathrm{II}}=\frac{\Gamma^{2}}{2 \Omega \mathscr{C}} J_{2}^{2},
$$

where the Casimir operator $\mathscr{C}$ and the (second) SU(1,1) generator $J_{2}$ are

$$
\mathscr{C}=\frac{1}{4 \Omega m}\left[\left(p_{1}^{2}-p_{2}^{2}\right)+m^{2} \Omega^{2}\left(x_{1}^{2}-x_{2}^{2}\right)\right]
$$

(taken to be positive) and

$$
J_{2}=\frac{m}{2}\left[\left(\dot{x}_{1} x_{2}-\dot{x}_{2} x_{1}\right)-\Gamma r^{2}\right]
$$

respectively, and $r$ is given by $r^{2}=x_{1}^{2}-x_{2}^{2}$.

Let us then impose the constraint $J_{2}|\Psi\rangle=0$, which defines physical states and guaranties that $H$ is bounded from below. This implies

$$
H|\Psi\rangle=H_{\mathrm{I}}|\Psi\rangle=2 \Omega \mathscr{C}|\Psi\rangle=\left(\frac{1}{2 m} p_{r}^{2}+\frac{K}{2} r^{2}\right)|\Psi\rangle,
$$

with $K \equiv m \Omega^{2}$. Hence, $H_{\mathrm{I}}$ reduces to the Hamiltonian for the two-dimensional isotropic (or radial) harmonic oscillator $\ddot{r}+\Omega^{2} r=0$. 
The physical states are invariant under time-reversal and periodical with period $\tau=2 \pi / \Omega$. The generic state $|\Psi(t)\rangle_{H}$ can be written as

$$
|\Psi(t)\rangle_{H}=\hat{T}\left[\exp \left(\frac{i}{\hbar} \int_{t_{0}}^{t} 2 \Gamma J_{2} d t^{\prime}\right)\right]|\Psi(t)\rangle_{H_{\mathrm{I}}}
$$

where $\hat{T}$ denotes time-ordering and the constant $\hbar$, with dimension of an action, is introduced for dimensional reasons. The states $|\Psi(t)\rangle_{H}$ and $|\Psi(t)\rangle_{H_{\mathrm{I}}}$ satisfy the equations:

$$
\begin{aligned}
i \hbar \frac{d}{d t}|\Psi(t)\rangle_{H} & =H|\psi(t)\rangle_{H}, \\
\text { and } \quad i \hbar \frac{d}{d t}|\Psi(t)\rangle_{H_{\mathrm{I}}} & =2 \Omega \mathscr{C}|\Psi(t)\rangle_{H_{\mathrm{I}}}
\end{aligned}
$$

respectively. The periodicity of the physical states imply

$$
\begin{aligned}
|\Psi(\tau)\rangle & =\exp \left(i \varphi-\frac{i}{\hbar} \int_{0}^{\tau}\langle\Psi(t)|H| \Psi(t)\rangle d t\right)|\Psi(0)\rangle \\
& =\exp (-i 2 \pi n)|\Psi(0)\rangle,
\end{aligned}
$$

or equivalently,

$$
\frac{\langle\Psi(\tau)|H| \Psi(\tau)\rangle}{\hbar} \tau-\varphi=2 \pi n, n=0,1,2, \ldots .
$$

Using $\tau=2 \pi / \Omega$ and $\varphi=\alpha \pi$, where $\alpha$ is a real constant, we thus obtain

$$
\left\langle\Psi_{n}(\tau)|H| \Psi_{n}(\tau)\right\rangle=\hbar \Omega\left(n+\frac{\alpha}{2}\right)
$$

The index $n$ signals the $n$ dependence of the state and the corresponding energy. Equation (3.30) gives the effective $n^{\text {th }}$ energy level of the system corrected by its interaction with the environment. In conclusion, the dissipation term $J_{2}$ of the Hamiltonian is responsible for the zero point $(n=0)$ energy $E_{0}=(\hbar / 2) \Omega \alpha$, which is the signature of quantization. In conclusion, the zero point quantum contribution $E_{0}$ to the spectrum of physical states signals the underlying dissipative dynamics.

\section{High Energy Phenomenology of the Noncommutative Spectral Geometry}

Let us proceed with a short discussion on the phenomenological consequences [1] of the noncommutative spectral approach to the standard model, the most successful particle physics model we have at hand.

As a consequence of the choice $M_{2}(\mathbb{H}) \oplus M_{4}(\mathbb{C})$ for the algebra $\mathscr{A}$ of the discrete space $\mathscr{F}$, the spectrum of the fermionic particles (the number of states in the Hilbert space) per family ${ }^{8}$ is predicted to be $4^{2}=16$. Moreover, the selected (in order to be consistent with the axioms of noncommutative geometry) algebra leads to the gauge group of the standard model. Thus, the 16 spinors get the correct quantum number with respect to the standard model gauge group. The gauge bosons of the standard model gauge group are the inner fluctuations of the metric along continuous directions. In addition, there is a Higgs doublet corresponding to the inner fluctuations

\footnotetext{
${ }^{8}$ The number of families is a physical input.
} 
along the discrete directions. The spectral action approach leads to a mass of this Higgs doublet with a negative sign and a quartic term with a plus sign, implying the existence of a mechanism of spontaneous breaking of the electroweak symmetry.

Let us assume that the function $f$ is well approximated by the cut-off function and ignore higher order terms. Normalization of the kinetic terms implies

$$
\frac{g_{3}^{2} f_{0}}{2 \pi^{2}}=\frac{1}{4} \text { and } g_{3}^{2}=g_{2}^{2}=\frac{5}{3} g_{1}^{2}
$$

leading to

$$
\sin ^{2} \theta_{\mathrm{W}}=\frac{3}{8}
$$

a relation which was also found in the context of $\mathrm{SU}(5)$ and $\mathrm{SO}(10)$ grand unified theories. Since the predicted relations, Eq. (4.1b), from the noncommutative spectral geometry are the ones that hold for all grand unified theories, this implies that the spectral action holds at unification scale.

Assuming the big desert hypothesis, the running of the couplings $\alpha_{i}=g_{i}^{2} /(4 \pi)$ with $i=1,2,3$, up to one-loop corrections ${ }^{9}$, is

$$
\beta_{i}=\frac{1}{(4 \pi)^{2}} b_{i} g_{i}^{3} \quad \text { with } \quad b=\left(\frac{41}{5},-\frac{19}{6},-7\right) .
$$

Performing one-loop renormalization group analysis for the running of the gauge couplings and the Newton constant, it was shown [1] that these do not meet at a point, the error being within just few percent. The fact that the predicted unification of the coupling constants does not hold exactly, implies that the big desert hypothesis is only approximately valid and new physics are expected between unification and present energy scales. In terms of our assumption for the cut-off function, the lack of a unique unification energy implies that even though the function $f$ can be approximated by the cut-off function there exist small deviations.

The noncommutative spectral geometry model predicts also the existence of a see-saw mechanism for neutrino masses with large right-handed neutrino mass of the order of $\Lambda$. Moreover, it predicts the constraint:

$$
\sum_{\sigma}\left(y_{v}^{\sigma}\right)^{2}+\left(y_{e}^{\sigma}\right)^{2}+3\left(y_{u}^{\sigma}\right)^{2}++3\left(y_{d}^{\sigma}\right)^{2}=4 g^{2}
$$

on the Yukawa couplings $y^{\sigma}$ with $\sigma=1,2,3$, at unification scale.

The mass of the top quark is given from

$$
m_{\mathrm{top}}=\frac{1}{\sqrt{2}} u k^{t}
$$

with $u=2 \mathrm{M} / \mathrm{g}$ the vacuum expectation value of the Higgs field and $k^{t}$ the top quark Yukawa coupling. We assume that at unification scale of $\sim 1.1 \times 10^{17} \mathrm{GeV}$ the value of $g$ is $\sim 0.517$ and the $\tau$ neutrino Yukawa coupling can be neglected. Then using the renormalization group equations, the model predicts a top quark mass of $\sim 179 \mathrm{GeV}$, compatible with the experimental value.

\footnotetext{
${ }^{9}$ Only at one-loop order the renormalization group equations for the coupling constants $g_{i}$ are uncoupled from the other standard model parameters.
} 
In the spectral action, the Higgs coupling is proportional to the gauge couplings, which restricts the mass of the Higgs. Using the cut-off function, this model predicts a heavy Higgs mass. In zeroth order approximation, it predicts a mass of the Higgs boson approximately equal to $170 \mathrm{GeV}$, which is ruled out by current experimental data. However, this answer is very sensitive to the value of the unification scale, as well as to deviations of the spectral function from the cut-off function we have used. The actual value of the Higgs mass will be determined by considering higher order corrections and incorporating them to the renormalization group equations. Nevertheless, it is quite encouraging that this purely geometric approach to the standard model predicted the right order of magnitude for the Higgs mass. Given that this noncommutative spectral geometry model has to be seen as an effective theory, this result is quite remarkable.

Since the predicted top quark mass is consistent with experimental data while the predicted Higgs mass is ruled out, one may deduce that the top quark mass is less sensitive to the ambiguities of the unification scale than the Higgs mass. This conclusion may be understood in the following way. We have splitted the action functional into the bosonic and the fermionic parts. The bosonic action has been then determined by an infinite expansion assuming convergence of higher order terms. Thus, while for the bosonic part we have relied on the first terms of the expansion in inverse powers of the cut-off scale, the fermionic part being much simpler did not require such an assumption.

Considering an energy scale $\Lambda \sim 1.1 \times 10^{17} \mathrm{GeV}$, the standard form of the gravitational action and the experimental value of Newton's constant at ordinary scales imply $\kappa_{0}^{-1} \sim 2.43 \times 10^{18} \mathrm{GeV}$.

Let us also note that this approach to unification does not provide any explanation of the number of generations, nor leads to constraints on the values of the Yukawa couplings.

Finally, the parameter $\Lambda$ which has been introduced as a free parameter in the spectral action, can be seen as the vacumm expectation value of a dynamical (dilaton) field. Such a filed may lead to cosmological consequences and it is worth examining whether it could play the rôle of the inflaton field.

\section{Cosmological consequences}

The noncommutative spectral action lives by construction at high energy scales, thus providing a natural framework to address early universe cosmology. Investigating the cosmological consequences of the model, one can test its validity and/or constrain its parameters. In what follows, we review some cosmological aspects of this purely geometric approach to the standard model. Let us first specify the notation and conventions we use. The signature is taken $(-,+,+,+)$ and the Ricci tensor is defined as $R_{\mu v}=R^{\rho}{ }_{\mu \nu \rho}$, with $R_{\mu v \rho}{ }^{\sigma} \omega_{\sigma}=[\nabla \mu, \nabla v] \omega_{\rho}$.

The Lorentzian version of the gravitational part of the asymptotic formula for the bosonic sector of the noncommutative geometry spectral action, including the coupling between the Higgs field and the Ricci curvature scalar, reads [1]

$$
\mathscr{S}_{\text {grav }}^{\mathrm{L}}=\int\left(\frac{1}{2 \kappa_{0}^{2}} R+\alpha_{0} C_{\mu v \rho \sigma} C^{\mu v \rho \sigma}+\tau_{0} R^{\star} R^{\star}-\xi_{0} R|\mathbf{H}|^{2}\right) \sqrt{-g} d^{4} x,
$$

leading to he equations of motion [6]

$$
R^{\mu v}-\frac{1}{2} g^{\mu v} R+\frac{1}{\beta^{2}} \delta_{\mathrm{cc}}\left[2 C_{; \lambda ; \kappa}^{\mu \lambda v \kappa}+C^{\mu \lambda v \kappa} R_{\lambda \kappa}\right]=\kappa_{0}^{2} \delta_{\mathrm{cc}} T_{\text {matter }}^{\mu v},
$$


where

$$
\beta^{2} \equiv-\frac{1}{4 \kappa_{0}^{2} \alpha_{0}} \quad \text { and } \quad \delta_{\mathrm{cc}} \equiv\left[1-2 \kappa_{0}^{2} \xi_{0} \mathbf{H}^{2}\right]^{-1} .
$$

Let us first study the low energy regime and then proceed with the high energy regime. Depending on whether we are in the former or the latter one, will specify whether or not the coupling between the Higgs field and the background geometry can be neglected.

\subsection{Low energy regime}

In the low energy weak curvature regime, the nonminimal coupling term between the background geometry and the Higgs field can be neglected, implying $\delta_{\mathrm{cc}}=1$. For a FriedmannLemaitre-Robertson-Walker space-time, the Weyl tensor vanishes, hence the noncommutative spectral geometry corrections to the Einstein equation vanish [6]. Thus for such a background, the constraint [23] $\beta_{R^{2}} \geq 3.2 \times 10^{-9} \mathrm{~m}^{-1}$, imposed on ad hoc curvature squared terms (of different form but of the same order) does not necessarily hold within the noncommutative spectral action context. It is however important to constrain $\beta$ since a lower limit to $\beta$ can be equivalently seen as an upper limit to the moment $f_{0}$ of the cut-off function used to define the spectral action. Since $f_{0}$ can be used to specify the initial conditions on the gauge couplings, a constraint on $\beta$ corresponds to a restriction on the particle physics model at unification scale.

Let us briefly summarize how one can constrain $\beta[9,10]$ within the noncommutative spectral geometry model. Consider linear perturbations around a Minkowski background metric in the synchronous gauge. The perturbed metric reads

$$
g_{\mu v}=\operatorname{diag}\left(\{a(t)\}^{2}\left[-1,\left(\delta_{i j}+h_{i j}(x)\right)\right]\right)
$$

with $a(t)$ the cosmological scale factor. Since we only consider a flat background, $a(t)=1$ and $\dot{a} \equiv d a / d t=0$. The remaining gauge freedom can be completely fixed by setting $\nabla_{i} h^{i j}=0$.

The linearized equations of motion derived from the noncommutative spectral action for such perturbations are

$$
\left(\square-\beta^{2}\right) \square h^{\mu v}=\beta^{2} \frac{16 \pi G}{c^{4}} T_{\text {matter }}^{\mu v},
$$

where $T_{\text {matter }}^{\mu v}$ is taken to lowest order in $h^{\mu v}$. It is thus independent of $h^{\mu v}$ and satisfies the conservation equations

$$
\frac{\partial}{\partial x^{\mu}} T_{v}^{\mu}=0 .
$$

Since $\beta$ plays the rôle of a mas, it has to be real and positive, implying $\alpha_{0}<0$. For $\alpha_{0}>0$ the gravitational waves evolve according to a Klein-Gordon like equation with a tachyonic mass, and hence the background, which has been considered to be a Minkowski space, is unstable. In conclusion, we must restrict to $\alpha_{0}<0$ for Minkowski space to be a (stable) vacuum of the theory.

Let us study the energy lost to gravitational radiation by orbiting binaries. In the far field limit, $|\mathbf{r}| \approx\left|\mathbf{r}-\mathbf{r}^{\prime}\right|$ (where $\mathbf{r}$ and $\mathbf{r}^{\prime}$ stand for the locations of the observer and emitter, respectively), the spatial components of the general first order solution for a perturbation against a Minkowski background are given in terms of the quadrupole moment,

$$
D^{i k}(t) \equiv \frac{3}{c^{2}} \int x^{i} x^{k} T^{00}(\mathbf{r}, t) d \mathbf{r} .
$$


as

$$
h^{i k}(\mathbf{r}, t) \approx \frac{2 G \beta}{3 c^{4}} \int_{-\infty}^{t-\frac{1}{c}|\mathbf{r}|} \frac{d t^{\prime}}{\sqrt{c^{2}\left(t-t^{\prime}\right)^{2}-|\mathbf{r}|^{2}}} \mathscr{J}_{1}\left(\beta \sqrt{c^{2}\left(t-t^{\prime}\right)^{2}-|\mathbf{r}|^{2}}\right) \ddot{D}^{i k}\left(t^{\prime}\right)
$$

where $\mathscr{J}_{1}$ is a Bessel function of the first kind, in terms of the quadrupole moment.

While in the $\beta \rightarrow \infty$ limit the theory reduces to that of General Relativity and the familiar results for a massless graviton are recovered, for finite $\beta$ gravitational radiation contains both massive and massless modes, both of which are sourced from the quadrupole moment of the system.

In the far field limit, the rate of energy loss for a binary pair of masses $m_{1}, m_{2}$ in a circular (for simplicity) orbit in the $(x y)$-plane, reads

$$
-\frac{\mathrm{d} \mathscr{E}}{\mathrm{d} t} \approx \frac{c^{2}}{20 G}|\mathbf{r}|^{2} \dot{h}_{i j} \dot{h}^{i j}
$$

with

$$
\dot{h}^{i j} \dot{h}_{i j}=\frac{128 \mu^{2}|\rho|^{4} \omega^{6} G^{2} \beta^{2}}{c^{8}}\left[f_{\mathrm{c}}^{2}\left(\beta|\mathbf{r}|, \frac{2 \omega}{\beta c}\right)+f_{\mathrm{s}}^{2}\left(\beta|\mathbf{r}|, \frac{2 \omega}{\beta c}\right)\right],
$$

and the definitions

$$
\begin{aligned}
& f_{\mathrm{c}}(x, z) \equiv \int_{0}^{\infty} \frac{d s}{\sqrt{s^{2}+x^{2}}} \mathscr{J}_{1}(s) \cos \left(z \sqrt{s^{2}+x^{2}}\right) \\
& f_{\mathrm{s}}(x, z) \equiv \int_{0}^{\infty} \frac{d s}{\sqrt{s^{2}+x^{2}}} \mathscr{J}_{1}(s) \sin \left(z \sqrt{s^{2}+x^{2}}\right)
\end{aligned}
$$

The orbital frequency $\omega$ is constant and given by

$$
\omega=|\rho|^{-3 / 2} \sqrt{G\left(m_{1}+m_{2}\right)}
$$

with $|\rho|$ the magnitude of the separation vector between the two bodies.

The integrals in Eq. (5.11) exhibit a strong resonance behavior at $z=1$, which corresponds to the critical frequency

$$
2 \omega_{\mathrm{c}}=\beta c
$$

around which strong deviations from the familiar results of General Relativity are expected. This critical (maximum) frequency comes from the natural length scale (given by $\beta^{-1}$ ), at which noncommutative geometry effects become dominant. For $\omega<\omega_{\mathrm{c}}$, the $\beta \rightarrow \infty$ limit reproduces the General Relativity result, as it should. Since this is not the case if $\omega>\omega_{\mathrm{c}}$, we conclude that the critical frequency is the maximum one. Any deviation from the standard result is suppressed by the distance to the source, at least for orbital frequencies small compared to $\beta c$.

The form of the gravitational radiation from binary systems can be used to constrain $\beta$. For circular binary orbits we only need to know the orbital frequency and the distance to the binary system. The parameter $\beta$ is then constrained by requiring the magnitude of deviations from General Relativity to be less than the uncertainty. Thus, for $\omega<\omega_{\mathrm{c}}$ we get a lower limit on $\beta$ [10]:

$$
\beta>7.55 \times 10^{-13} \mathrm{~m}^{-1}
$$


Due to the large distances to these binary systems, the constraint is almost exactly due to $\beta>$ $2 \omega / c$. Thus, the strongest constraint comes from systems with high orbital frequencies. Future observations of rapidly orbiting binaries, relatively close to the Earth, could thus improve this constraint by many orders of magnitude.

Let us go back to the background equations. In order for the corrections to Einstein's equations to be apparent at the level of the background, we need to consider anisotropic models. We will thus derive the modified Friedmann equation for the Bianchi type- $\mathrm{V}$ model, for which the space-time metric in Cartesian coordinates reads

$$
g_{\mu v}=\operatorname{diag}\left[-1,\left\{a_{1}(t)\right\}^{2} e^{-2 n z},\left\{a_{2}(t)\right\}^{2} e^{-2 n z},\left\{a_{3}(t)\right\}^{2}\right]
$$

where $a(t), b(t)$ and $c(t)$ are, in general, arbitrary functions and $n$ is an integer.

Defining $A_{i}(t)=\ln a_{i}(t)$ with $i=1,2,3$, the modified Friedmann equation reads [6]:

$$
\begin{array}{r}
\kappa_{0}^{2} T_{00}= \\
-\dot{A}_{3}\left(\dot{A}_{1}+\dot{A}_{2}\right)-n^{2} e^{-2 A_{3}}\left(\dot{A}_{1} \dot{A}_{2}-3\right) \\
+\frac{8 \alpha_{0} \kappa_{0}^{2} n^{2}}{3} e^{-2 A_{3}}\left[5\left(\dot{A}_{1}\right)^{2}+5\left(\dot{A}_{2}\right)^{2}-\left(\dot{A}_{3}\right)^{2}\right. \\
\left.-\dot{A}_{1} \dot{A}_{2}-\dot{A}_{2} \dot{A}_{3}-\dot{A}_{3} \dot{A}_{1}-\ddot{A}_{1}-\ddot{A}_{2}-\ddot{A}_{3}+3\right]-\frac{4 \alpha_{0} \kappa_{0}^{2}}{3} \sum_{i}\left\{\dot{A}_{1} \dot{A}_{2} \dot{A}_{3} \dot{A}_{i}\right. \\
+\dot{A}_{i} \dot{A}_{i+1}\left(\left(\dot{A}_{i}-\dot{A}_{i+1}\right)^{2}-\dot{A}_{i} \dot{A}_{i+1}\right)+\left(\ddot{A}_{i}+\left(\dot{A}_{i}\right)^{2}\right)\left[-\ddot{A}_{i}-\left(\dot{A}_{i}\right)^{2}+\frac{1}{2}\left(\ddot{A}_{i+1}+\ddot{A}_{i+2}\right)\right. \\
\left.+\frac{1}{2}\left(\left(\dot{A}_{i+1}\right)^{2}+\left(\dot{A}_{i+2}\right)^{2}\right)\right]+\left[\dddot{A}_{i}+3 \dot{A}_{i} \ddot{A}_{i}-\left(\ddot{A}_{i}+\left(\dot{A}_{i}\right)^{2}\right)\left(\dot{A}_{i}-\dot{A}_{i+1}-\dot{A}_{i+2}\right)\right] \\
\left.\times\left[2 \dot{A}_{i}-\dot{A}_{i+1}-\dot{A}_{i+2}\right]\right\} .
\end{array}
$$

The correction terms in this modified Friedmann equation come in two types. The first one contains terms which are fourth order in time derivatives, and thus for the slowly varying functions, usually used in cosmology, they can be taken to be small corrections. The second one occurs at the same order as the standard Einstein-Hilbert terms, however being proportional to $n^{2}$, it vanishes for homogeneous versions of Bianchi type-V. Thus, although anisotropic cosmologies do contain corrections due to the additional noncommutative spectral geometry terms in the action, they are typically of higher order. Inhomogeneous models do contain correction terms that appear on the same footing as the original (commutative) terms. In conclusion, the corrections to Einstein's equations are present only in inhomogeneous and anisotropic space-times.

\subsection{High energy regime}

At energies approaching the Higgs scale, the nonminimal coupling of the Higgs field to the curvature can no longer be neglected, leading to corrections even for background cosmologies. To 
understand the effects of these corrections let us neglect the conformal term in Eq. (5.2), i.e. set $\beta=0$. The equations of motion then become [6]

$$
R^{\mu v}-\frac{1}{2} g^{\mu v} R=\kappa_{0}^{2}\left[\frac{1}{1-\kappa_{0}^{2}|\mathbf{H}|^{2} / 6}\right] T_{\text {matter }}^{\mu v} .
$$

Hence, $|\mathbf{H}|$ leads to an effective gravitational constant.

Alternatively, consider the effect of this term on the equations of motion for the Higgs field in a constant gravitational field. For constant curvature, the self interaction of the Higgs field is increased, since

$$
-\mu_{0}|\mathbf{H}|^{2} \rightarrow-\left(\mu_{0}+\frac{R}{12}\right)|\mathbf{H}|^{2}
$$

The nonminimal coupling between the Higgs field and the Ricci curvature may turn out to be particularly useful in early universe cosmology [7,8]. Such a coupling has been introduced ad hoc in the literature, in an attempt to drive inflation through the Higgs field. However, the coupling constant between the scalar field and the background geometry is not a free parameter which could be tuned to achieve a successful inflationary scenario, it should be instead dictated by the underlying theory.

In a Friedmann-Lemaittre-Robertson-Walker metric, the Weyl tensor vanishes, while the nondynamical term is also neglected. Thus the Gravity-Higgs sector of the asymptotic expansion of the spectral action, in Lorentzian signature reads

$$
\mathscr{S}_{\mathrm{GH}}^{\mathrm{L}}=\int\left[\frac{1-2 \kappa_{0}^{2} \xi_{0} H^{2}}{2 \kappa_{0}^{2}} R-\frac{1}{2}(\nabla H)^{2}-V(H)\right] \sqrt{-g} d^{4} x,
$$

where

$$
V(H)=\lambda_{0} H^{4}-\mu_{0}^{2} H^{2},
$$

with $\mu_{0}$ and $\lambda_{0}$ subject to radiative corrections as functions of energy. For large enough values of the Higgs field, the renormalized value of these parameters must be calculated, while the running of the top Yukawa coupling and the gauge couplings must be evolved simultaneously.

At high energies the mass term is sub-dominant and can be neglected, thus only the first term in Eq. (5.19) survives. For each value of the top quark mass, there is a value of the Higgs mass where the effective potential is on the verge of developing a metastable minimum at large values of the Higgs field and the Higgs potential is locally flattened [8]. Since the region where the potential becomes flat is narrow, slow-roll must be very slow, in order to provide a sufficiently long period of quasi-exponential expansion and thus solve the shortcomings of the standard hot big bang cosmological model. If the inflaton field is also going to source the initial density fluctuations then besides the constraints on the slow-roll parameters $\varepsilon, \eta$ to get sufficient number of e-foldings, one should also check whether the amplitude of density perturbations $\Delta_{\mathscr{R}}^{2}$ in the spectrum of the cosmic microwave background temperature anisotropies is in agreement with measurements. Inflation predicts that at horizon crossing (denoted by stars), the amplitude of density perturbations is related to the inflaton potential $V$ through

$$
\left(\frac{V_{*}}{\varepsilon_{*}}\right)^{\frac{1}{4}}=2 \sqrt{3 \pi} m_{\mathrm{Pl}} \Delta_{\mathscr{R}}^{\frac{1}{2}},
$$


where $\varepsilon_{*} \leq 1$ and $m_{\mathrm{Pl}}$ stands for the Planck mass. Its value, as measured by WMAP7 [24], requires

$$
\left(\frac{V_{*}}{\varepsilon_{*}}\right)^{\frac{1}{4}}=(2.75 \pm 0.30) \times 10^{-2} m_{\mathrm{Pl}} .
$$

We can then calculate the renormalization of the Higgs self-coupling and construct an effective potential which fits the renormalization group improved potential around the flat region. By doing this calculation up to two-loops we have found [8] that around the plateau (the minimum of the potential), there is a very good analytic fit to the Higgs potential, which takes the form

$$
V^{\mathrm{eff}}=\lambda_{0}^{\mathrm{eff}}(H) H^{4}=\left[a \ln ^{2}(b \kappa H)+c\right] H^{4},
$$

where the parameters $a, b$ are found to relate to the low energy values of top quark mass $m_{\mathrm{t}}$ as

$$
\begin{aligned}
& a\left(m_{\mathrm{t}}\right)=4.04704 \times 10^{-3}-4.41909 \times 10^{-5}\left(\frac{m_{\mathrm{t}}}{\mathrm{GeV}}\right)+1.24732 \times 10^{-7}\left(\frac{m_{\mathrm{t}}}{\mathrm{GeV}}\right)^{2}, \\
& b\left(m_{\mathrm{t}}\right)=\exp \left[-0.979261\left(\frac{m_{\mathrm{t}}}{\mathrm{GeV}}-172.051\right)\right] .
\end{aligned}
$$

The third parameter, $c=c\left(m_{\mathrm{t}}, m_{\phi}\right)$, encodes the appearance of an extremum and depends on the values for top quark mass and Higgs mass. An extremum occurs if and only if $c / a \leq 1 / 16$, the saturation of the bound corresponding to a perfectly flat region. It is convenient to write $c=$ $[(1+\delta) / 16] a$, where $\delta=0$ saturates the bound below which a local minimum is formed.

These results have been obtained for the case of minimal coupling, whereas in noncommutative spectral action there is a small nonminimal coupling, $\xi_{0}=1 / 12$. The corrections due to conformal coupling to the potential imply that flatness does not occur at $\delta=0$ anymore but for fixed values of $\delta$ depending on the value of the top quark mass. More precisely, for inflation to occur via this mechanism, the top quark mass fixes the Higgs mass extremely accurately. Scanning through the parameter space it emerges that sufficient $e$-folds are indeed generated provided there is a suitably tuned relationship between the top quark mass and the Higgs mass. In conclusion, while the Higgs potential can lead to the slow-roll conditions being satisfied once the running of the selfcoupling at two-loops is included, the constraints imposed from the cosmic microwave background temperature anisotropies measurements make the predictions of such a scenario incompatible with the measured value of the top quark mass.

Finally, running of the gravitational constant and corrections by considering the more appropriate de Sitter, instead of a Minkowski, background do not improve substantially the realization of a successful inflationary era [8].

The noncommutative spectral action provides in addition to the Higgs field, another (massless) scalar field [25], denoted by $\sigma$, which is unlike all other fields in the theory, such as the Higgs field and gauge fields. Note that $\sigma$ does not exhibit a coupling to the matter sector.

Including this field, the cosmologically relevant terms in the Wick rotated action read [25]

$$
\mathscr{S}=\int\left[\frac{1}{2 \kappa^{2}} R-\xi_{H} R H^{2}-\xi_{\sigma} R \sigma^{2}-\frac{1}{2}(\nabla H)^{2}-\frac{1}{2}(\nabla \sigma)^{2}-V(H, \sigma)\right] \sqrt{-g} d^{4} x,
$$


where

$$
V(H, \sigma)=\lambda_{H} H^{4}-\mu_{H}^{2} H^{2}+\lambda_{\sigma} \sigma^{4}+\lambda_{H \sigma}|H|^{2} \sigma^{2} .
$$

The constants are related to the underlying parameters as follows:

$$
\begin{aligned}
\xi_{H} & =\frac{1}{12}, & \xi_{\sigma} & =\frac{1}{12} \\
\lambda_{H} & =\frac{\pi^{2} \mathfrak{b}}{2 f_{0} \mathfrak{a}^{2}}, & \lambda_{\sigma} & =\frac{\pi^{2} \mathfrak{d}}{f_{0} \mathfrak{c}^{2}} \\
\mu_{H} & =2 \Lambda^{2} \frac{f_{2}}{f_{0}}, & \lambda_{H \sigma} & =\frac{2 \pi^{2} \mathfrak{e}}{a \mathfrak{c} f_{0}} .
\end{aligned}
$$

In conclusion, neither the $\sigma$ field can lead to a successful slow-roll inflationary era, if the coupling values are conformal [8].

One should then examine whether the dilaton field, a dynamical field that can replace the cutoff $\Lambda$, could play the rôle of the inflaton ${ }^{10}$. Then the operator $\mathscr{D} / \Lambda$ is replaced by $e^{-\Phi / 2} \mathscr{D} e^{-\Phi / 2}$, where $\Phi$ stands for the dilaton field [18]. The action for the Gravity-Dilaton-Higgs sectors, was then shown to be [18]

$$
\begin{gathered}
\mathscr{S}_{\mathrm{GDH}}=\int \sqrt{G}\left[-\frac{1}{2 \kappa_{0}^{2}} R+\frac{1}{2}\left(1+\frac{6}{\kappa_{0}^{2} f^{2}}\right) G^{\mu v} \partial_{\mu} \Phi \partial_{v} \Phi\right. \\
\left.+G^{\mu v} D_{\mu} H^{*} D_{v} H^{\prime}-V_{0}\left(H^{*} H^{\prime}\right)\right] d^{4} x,
\end{gathered}
$$

where $G_{\mu \nu}$ is the metric in Einstein frame and $f$ stands for the dilaton decay constant. The scale $f$ is of the order of the Planck scale. The dilaton $\Phi$ can be related to a scalar field $\tilde{\sigma}$ of dimension one through $\Phi=(1 / f) \tilde{\sigma}$. It is worth noting that the difference between the above action and the spectral one is that the latter has a conformal coupling between the background geometry, in other words the Ricci curvature $R(G)$, and the Higgs field $H$, which is required in order to get scale invariant matter couplings.

Certainly, to investigate whether the dilaton field $\Phi$ could play the rôle of the inflaton, one should first calculate its potential.

\section{Conclusions}

In Connes' formulation of noncommutative geometry, which we have adopted here, mathematical and physical notions are described in terms of spectral properties of operators. By extending the one-to-one correspondence between spaces and commutative $C^{\star}$-algebras to noncommutative algebras, Connes' approach aims at mapping notions of differential geometry into algebraic terms. The topology of space is described in terms of the algebras, or equivalently, the properties of space are encoded in some continuous fields. The model depends crucially on the choice of the algebra $\mathscr{A}$, represented on a Hilbert space $\mathscr{H}$, and the generalized Dirac operator $\mathscr{D}$. These $\mathscr{A}, \mathscr{H}, \mathscr{D}$ form a spectral triple, a fundamental ingredient of the whole formalism, which contains the information on the geometry of space-time. The Dirac operator $\mathscr{D}$ describes the metric aspects of the model and the behavior of the fundamental matter fields represented by vectors of the Hilbert space $\mathscr{H}$.

\footnotetext{
${ }^{10}$ In particular on non-compact spaces where the Dirac operator has no longer a discrete spectrum.
} 
The fluctuations of the Dirac operator $\mathscr{D}$ contain the boson fields, including the mediators of the forces and the Higgs field.

This noncommutative spectral geometry model has been introduced as an approach to the standard model of particle physics coupled to gravity. By considering the standard model of strong and electroweak interactions as a phenomenological model, one tries to retrieve the noncommutative geometry of space-time. It turns out that the geometry can be considered as the product $\mathscr{M} \times \mathscr{F}$ of a four-dimensional smooth compact Riemanian manifold $\mathscr{M}$ by a discrete finite noncommutative space $\mathscr{F}$ composed of just two points. The choice of the discrete space is specified by the symmetries of the Hilbert space in which quarks and leptons are placed.

The physical picture is straightforward. The left- and right-handed fermions are placed on two different sheets. The Higgs fields are just the gauge fields in the discrete dimensions. The inverse of the separation between the two sheets can be interpreted as the electroweak energy scale. It is interesting to remark that this picture is similar to the the Randall-Sundrum scenario, where a four-dimensional brane is embedded into a five-dimensional manifold as a three-dimensional brane placed at $x_{5}=0$ and $x_{5}=\pi r_{\text {comp }}$, with $r_{\text {comp }}$ the compactification radius.

The choice of a discrete space consisting of only two points can be (naively) criticized as a simplified approach. However, the two-sheeted construction has a deeper physical meaning. The doubling of the algebra is related to dissipation and the gauge field structure, required to explain the standard model of particle physics. Moreover, by applying 't Hooft's conjecture, stating that loss of information within completely deterministic dynamics can lead to a quantum evolution, dissipation may then lead to quantum features. Thus, the classical construction of noncommutative spectral geometry carries implicit in the doubling of the algebra the seeds of quantization.

The noncommutative spectral geometry model lives by construction at very high energy scales. It hence provides a natural framework to study early universe cosmology. In other words, it motivates a particular gravitational model which applied to a given cosmological background can lead to interesting observational consequences.

It is a pleasure to thank the organizers of the Workshop on Non Commutative Field Theory and Gravity, held in the beautiful island of Corfu, for inviting me to present this work during a stimulating and interesting meeting.

\section{References}

[1] A. H. Chamseddine, A. Connes and M. Marcolli, Gravity and the standard model with neutrino mixing, Adv. Theor. Math. Phys. 11, 991 (2007) [arXiv:hep-th/0610241].

[2] A. Connes, Noncommutative Geometry, Academic Press, New York (1994).

[3] A. Connes and M. Marcolli, Noncommutative Geometry, Quantum Fields and Motives, Hindustan Book Agency, India (2008).

[4] M. Sakellariadou, A. Stabile and G. Vitiello G, Noncommutative spectral geometry, algebra doubling and the seeds of quantization, Phys. Rev. D 84045026 (2011) [arXiv:1106.4164 [math-ph]]. 
[5] M. Sakellariadou, Noncommutative Geometry Spectral Action as a framework for unification: Introduction and phenomenological/cosmological consequences, Int. J. Mod. Phys. D 20785 (2011) [arXiv:1008.5348 [hep-th]].

[6] W. Nelson and M. Sakellariadou, Cosmology and the Noncommutative approach to the Standard Model, Phys. Rev. D 81, 085038 (2010) [arXiv:0812.1657 [hep-th]].

[7] W. Nelson and M. Sakellariadou, Natural inflation mechanism in asymptotic noncommutative geometry, Phys. Lett. B 680, 263 (2009) [arXiv:0903.1520 [hep-th]].

[8] M. Buck, M. Fairbairn and M. Sakellariadou, Inflation within models with Conformal Coupling between the Scalar field and the Ricci Curvature: An application to the Noncommutative Spectral Action, Phys. Rev. D 82043509 (2010) [arXiv:1005.1188 [hep-th]].

[9] W. Nelson, J. Ochoa and M. Sakellariadou, Gravitational Waves in the Spectral Action of Noncommutative Geometry, Phys. Rev. D 82 (2010) 085021 [arXiv:1005.4276 [hep-th]].

[10] W. Nelson, J. Ochoa and M. Sakellariadou, Constraining the Noncommutative Spectral Action via Astrophysical Observations, Phys. Rev. Lett. 105 (2010) 101602 [arXiv:1005.4279 [hep-th]].

[11] A. Connes, Noncommutative Geometry and the standard model with neutrino mixing, JHEP 0611,081 (2006) [arXiv:hep-th/0608226].

[12] J. Barrett, A Lorentzian version of the non-commutative geometry of the standard model of particle physics, J. Math. Phys. 48, 012303 (2007) [arXiv:hep-th/060822].

[13] A. H. Chamseddine, A. Connes, Why the standard model, J. Geom. Phys. 58, 38 (2008) [arXiv:0706.3688 [hep-th]].

[14] A. H. Chamseddine and A. Connes, Noncommutative Geometry as a Framework for Unification of all Fundamental Interactions including Gravity. Part I, Fortschritte der Physik 58553 (2010) [arXiv:1004.0464 [hep-th]].

[15] A. H. Chamseddine and A. Connes, Conceptual Explanation for the Algebra in the Noncommutative Approach to the Standard Model, hys. Rev. Lett. 99, 191601 (2007) [arXiv:0706.3690 [hep-th]].

[16] A. H. Chamseddine and A. Connes, A Universal Action Formula, Phys. Rev. Lett. 77, 4868 (1996) [arXiv:hep-th/9606056].

[17] A. H. Chamseddine and A. and Connes The Spectral Action Principle, Commun. Math. Phys. 186731 (1997) [arXiv:hep-th/9606001].

[18] A. H. Chamseddine and A. and Connes, Scale Invariance in the Spectral Action, J. Math. Phys. 47 063504 (2006) [arXiv:hep-th/0512169].

[19] M. Blasone, Y. N. Srivastava, G. Vitiello and A. Widom, Phase coherence in quantum Brownian motion, Annals Phys. 267, 61 (1998) [arXiv:quant-ph/9707048].

[20] G. 't Hooft, Quantum Gravity as a Dissipative Deterministic System, Class. Quant. Grav. 163263 (1999) [arXiv:gr-qc/9903084].

[21] M. Blasone, P. Jizba and G. Vitiello, Dissipation and quantization, Phys. Lett. A 287205 (2001) [arXiv:hep-th/0007138].

[22] M. Blasone, E. Graziano, O. K. Pashaev and G. Vitiello G, Dissipation and Topologically Massive Gauge Theories in Pseudoeuclidean Plane Annals Phys. 252115 (1996) [arXiv:hep-th/9603092].

[23] K. S. Steele, Classical Gravity with Higher Derivatives, Gen. Rel. Grav. 9353 (1978). 
[24] D. Larson et al., Seven-Year Wilkinson Microwave Anisotropy Probe (WMAP) Observations: Are There Cosmic Microwave Background Anomalies?, Astrophys. J. Suppl. 19217 (2011) [arXiv:1001.4758 [astro-ph]].

[25] A. H. Chamseddine, Noncommutative Geometry as the Key to Unlock the Secrets of Space-Time, To be published in the Proceedings series of the Clay Mathematics Institute, eds. E. Blanchard et al [arXiv:0901.0577 [hep-th]]. 